In Soo Baek, Pusan University of Foreign Studies, Pusan 608-738, Korea.

e-mail: isbaek@taejo.pufs.ac.kr

\title{
WEAK LOCAL DIMENSION ON DERANGED CANTOR SETS
}

\begin{abstract}
We define weak local dimension at each point in a deranged Cantor set. Using this, we classify the deranged Cantor set into a multifractal structure which is useful for computing the Hausdorff dimension and the packing dimension of the deranged Cantor set. Finally, we give examples in which the weak local dimensions are used for the calculation of the dimensions in a deterministic case and a probabilistic case.
\end{abstract}

\section{Introduction}

The theory of a multifractal spectrum or a local dimension ([5]) of a fractal has been studied because it is important to investigate its geometric structure and fractal dimension. In fact, we need a nice mass distribution which represents the geometric structure of a given fractal well to discover the exact value of its fractal dimension. However it is difficult to find such a nice mass distribution on the fractal and it is also hard to compute the local dimension at each point in the fractal with respect to the mass distribution. It is also difficult for the case of a generalized Cantor set which we call a deranged Cantor set ([2], [3]). In this paper, we use a simple definition of weak local dimension at each point in a deranged Cantor set to avoid such difficulties. In fact, we don't use any mass distribution on a deranged Cantor set but its construction structure to define the weak local dimension. Ultimately the weak local dimension may be related to some natural mass distribution but it is open. To define weak local dimension, we need to define some terminologies such as local Hausdorff measure, local Hausdorff dimension, local packing measure, local packing dimension. We call local Hausdorff (packing) dimension at a point, weak lower (upper) local dimension at the point.

Key Words: weak local dimension, deranged Cantor set, Hausdorff dimension, and packing dimension

Mathematical Reviews subject classification: Primary 28A80, Secondary 60B05

Received by the editors October 29, 1999 
Now we recall the definition of deranged Cantor set ([2], [3]). Let $I_{\phi}=$ $[0,1]$. Then we obtain the left subinterval $I_{\tau, 1}$ and the right subinterval $I_{\tau, 2}$ of $I_{\tau}$ by deleting the middle open subinterval of $I_{\tau}$ inductively for each $\tau \in$ $\{1,2\}^{n}$, where $n=0,1,2, \ldots$ Consider $E_{n}=\bigcup_{\tau \in\{1,2\}^{n}} I_{\tau}$. Then $\left\{E_{n}\right\}$ is a decreasing sequence of closed sets. For each $n$, we put $\left|I_{\tau, 1}\right| /\left|I_{\tau}\right|=c_{\tau, 1}$ and $\left|I_{\tau, 2}\right| /\left|I_{\tau}\right|=c_{\tau, 2}$ for all $\tau \in\{1,2\}^{n}$, where $|I|$ denotes the diameter of $I$. We call $F=\bigcap_{n=0}^{\infty} E_{n}$ a deranged Cantor set.

We note that if $x \in F$, then there is $\sigma \in\{1,2\}^{N}$ such that $\bigcap_{k=0}^{\infty} I_{\sigma \mid k}=\{x\}$ (Here $\sigma \mid k=i_{1}, i_{2}, \cdots, i_{k}$ where $\sigma=i_{1}, i_{2}, \ldots, i_{k}, i_{k+1}, \ldots$ ). Hereafter, we identify $x \in F$ and the corresponding $\sigma \in\{1,2\}^{N}$. We define

$$
h^{s}(\sigma)=\liminf _{k \rightarrow \infty}\left(c_{1}^{s}+c_{2}^{s}\right)\left(c_{\sigma \mid 1,1}^{s}+c_{\sigma \mid 1,2}^{s}\right)\left(c_{\sigma \mid 2,1}^{s}+c_{\sigma \mid 2,2}^{s}\right) \ldots\left(c_{\sigma \mid k, 1}^{s}+c_{\sigma \mid k, 2}^{s}\right)
$$

for each $\sigma \in\{1,2\}^{N}$, and call $h^{s}(\sigma)$ the $s$-dimensional local Hausdorff measure of $\sigma$ in $F$.

We define $f(\sigma)=\inf \left\{s>0: h^{s}(\sigma)=0\right\}$ and call it the local Hausdorff dimension of $\sigma$ in $F$. Clearly we see that $f(\sigma)=\sup \left\{s>0: h^{s}(\sigma)=\infty\right\}$

Dually we define the local packing measure $q^{s}(\sigma)$ of $\sigma$ in $F$, and the local packing dimension $g(\sigma)$ of $\sigma$ in $F$. To be precise, for $\sigma \in F$

$$
\begin{aligned}
q^{s}(\sigma) & =\limsup _{k \rightarrow \infty}\left(c_{1}^{s}+c_{2}^{s}\right)\left(c_{\sigma \mid 1,1}^{s}+c_{\sigma \mid 1,2}^{s}\right)\left(c_{\sigma \mid 2,1}^{s}+c_{\sigma \mid 2,2}^{s}\right) \cdots\left(c_{\sigma \mid k, 1}^{s}+c_{\sigma \mid k, 2}^{s}\right), \\
g(\sigma) & =\inf \left\{s>0: q^{s}(\sigma)=0\right\}=\sup \left\{s>0: q^{s}(\sigma)=\infty\right\}
\end{aligned}
$$

We call the local Hausdorff (packing) dimension of $\sigma$ in $F$, the weak lower (upper) local dimension of $\sigma$ in $F$ compared with a local dimension of $\sigma$ in $F$ with respect to some mass distribution.

To compare the weak local dimension of $\sigma$ in $F$ with a local dimension of $\sigma$ in $F$ with respect to a mass distribution, we will consider the most related mass distribution $\mu_{s}$. We define

$$
\mu_{s}\left(I_{\tau}\right)=\frac{\left|I_{\tau}\right|^{s}}{\left(c_{1}^{s}+c_{2}^{s}\right)\left(c_{i_{1}, 1}^{s}+c_{i_{1}, 2}^{s}\right) \cdots\left(c_{i_{1}, i_{2}, \cdots, i_{k-1}, 1}^{s}+c_{i_{1}, i_{2}, \ldots, i_{k-1}, 2}^{s}\right)}
$$

for each $\tau=i_{1}, i_{2}, \cdots, i_{k-1}, i_{k}$, where $i_{j} \in\{1,2\}$. Then $\mu_{s}$ is extended to a Borel measure on $F$ for each $s \in(0,1)$ and $\mu_{s}(F)=1$ (see [4], [5]).

Now we can think of a multifractal structure $E_{s}, G_{s}$ on $F$ using weak local dimensions, $E_{s}=\{\sigma \in F: f(\sigma)=s\}$ and $G_{s}=\{\sigma \in F: g(\sigma)=s\}$. Then $F$ 
is classified as $F=\bigcup_{0<s<1} E_{s}$ and $F=\bigcup_{0<s<1} G_{s}$. We conjecture

$$
\begin{aligned}
E_{s} & =\left\{x \in F: \liminf _{r \rightarrow 0} \frac{\log \mu_{s}\left(B_{r}(x)\right)}{\log r}=s\right\} \\
G_{s} & =\left\{x \in F: \limsup _{r \rightarrow 0} \frac{\log \mu_{s}\left(B_{r}(x)\right)}{\log r}=s\right\} .
\end{aligned}
$$

\section{Main Results}

In this section, $F$ means a deranged Cantor set determined by $\left\{c_{\tau}\right\}$ with $\tau \in\{1,2\}^{n}$ where $n=1,2, \ldots$. Hereafter we only consider a deranged Cantor set whose contraction ratios $\left\{c_{\tau}\right\}$ and gap ratios $d_{\tau}\left(=1-\left(c_{\tau, 1}+c_{\tau, 2}\right)\right)$ are uniformly bounded away from 0 .

Before going into our main theorem, we note that the function $h^{s}(\sigma)$ or $h^{s}(x)$ is clearly a Borel function from $F$ to the extended real numbers. Given $s>0$, let $A_{s}=\bigcup_{t \leq s} E_{t}=\bigcap_{n=1}^{\infty}\left\{\sigma: h^{s+\frac{1}{n}}(\sigma)=0\right\}$. Then $A_{s}$ is a Borel set. Thus $E_{s}=A_{s} \backslash \bigcup_{n=1}^{\infty} A_{s-\frac{1}{n}}$ is a Borel set for each $s$. Since $A_{s}=\{\sigma: f(\sigma) \leq s\}$ is a Borel set, $f$ is a Borel function and hence $\mu_{s}$-measurable for all $s$.

Theorem 1. If $\mu_{s}\left(\bigcup_{t>s} E_{t}\right)>0$, then $\operatorname{dim}_{H}\left(\bigcup_{t>s} E_{t}\right) \geq s$.

Proof. Assume that $c_{\tau}, d_{\tau} \geq \alpha>0$ for some small $\alpha$. Let $x \in F$. Then there is unique $\sigma \in\{1,2\}^{N}$ such that $\bigcap_{k=0}^{\infty} I_{\sigma \mid k}=\{x\}$. Assume that $\mu_{s}\left(\bigcup_{t>s} E_{t}\right)>$ 0 . Let $\sigma(\equiv x) \in \bigcup_{t>s} E_{t}$. Given a small positive number $r$, there exists $k$ such that $\left|I_{\sigma \mid k+1}\right| \leq r<\left|I_{\sigma \mid k}\right|$. Since $d_{\sigma \mid j}\left|I_{\sigma \mid j}\right| \geq \alpha\left|I_{\sigma \mid k}\right|>\alpha r$ for $0 \leq j \leq k$, $B_{\alpha r}(x) \subset\left[\bigcup_{\tau(\neq \sigma \mid k) \in\{1,2\}^{k}} I_{\tau}\right]^{c}$, where $B_{\alpha r}(x)$ is the ball of radius $\alpha r$ with center $x$. Thus $\mu_{s}\left(B_{\alpha r}(x)\right) \leq \mu_{s}\left(I_{\sigma \mid k}\right)$.

$$
\limsup _{r \rightarrow 0} \frac{\mu_{s}\left(B_{r}(x)\right)}{r^{s}} \leq \frac{1}{\alpha^{2 s} h^{s}(\sigma)}=0 .
$$

Since $\mu_{s}\left(\bigcup_{t>s} E_{t}\right)>0, H^{s}\left(\bigcup_{t>s} E_{t}\right)=\infty$ ([5, Prop. 2.2 (a) $\left.]\right)$. Consequently, $\operatorname{dim}_{H}\left(\bigcup_{t>s} E_{t}\right) \geq s$.

Corollary 2. $\operatorname{dim}_{H}(F) \geq \sup \left\{s>0: \mu_{s}\left(\bigcup_{t>s} E_{t}\right)>0\right\}$.

Proof. Let $t^{\prime}<\sup \left\{s>0: \mu_{s}\left(\bigcup_{t \geq s} E_{t}\right)>0\right\}$. Then there exists $s$ such that $t^{\prime}<s \leq \sup \left\{s>0: \mu_{s}\left(\bigcup_{t>s} E_{t}\right)>0\right\}$ and $\mu_{s}\left(\bigcup_{t>s} E_{t}\right)>0$. So, by Theorem $1, \operatorname{dim}_{H}\left(\bigcup_{t>s} E_{t}\right) \geq s$. Hence $\operatorname{dim}_{H}(F)>t^{\prime}$.

Corollary 3. $\operatorname{dim}_{H}\left(\bigcup_{t \leq s} E_{t}\right) \leq s$. 
Proof. Let $t^{\prime}>s$ and $\sigma(\equiv x) \in \bigcup_{t \leq s} E_{t}$. Given a small positive number $r$, there exists $k$ such that $\left|I_{\sigma \mid k+1}\right| \leq r<\left|I_{\sigma \mid k}\right|$. Then $\frac{\mu_{t^{\prime}}\left(B_{r}(x)\right)}{r^{t^{\prime}}} \geq \frac{\mu_{t^{\prime}}\left(I_{\sigma \mid k+1}\right)}{\left|I_{\sigma \mid k}\right|^{t^{\prime}}}$. Since $\left|I_{\sigma \mid k+1}\right| /\left|I_{\sigma \mid k}\right|=c_{\sigma \mid k+1}>\alpha$ for all $k$,

$$
\limsup _{r \rightarrow 0} \frac{\mu_{t^{\prime}}\left(B_{r}(x)\right)}{r^{t^{\prime}}} \geq \frac{\alpha^{t^{\prime}}}{h^{t^{\prime}}(\sigma)}=\infty .
$$

Since $\mu_{t^{\prime}}\left(\bigcup_{t \leq s} E_{t}\right) \leq \mu_{t^{\prime}}(F)=1, H^{t^{\prime}}\left(\bigcup_{t \leq s} E_{t}\right)=0$. So $\operatorname{dim}_{H}\left(\bigcup_{t \leq s} E_{t}\right) \leq$ $t^{\prime}$.

Corollary 4. Let $s_{\tau}$ be the solution of $c_{\tau, 1}^{s_{\tau}}+c_{\tau, 2}^{s_{\tau}}=1$. If for any $t<s$, $\mu_{t}\left(\left\{\sigma \in\{1,2\}^{N}: \liminf _{k \rightarrow \infty} s_{\sigma \mid k} \geq s\right\}\right)>0$, then

$$
\operatorname{dim}_{H}\left(\left\{\sigma \in\{1,2\}^{N}: \liminf _{k \rightarrow \infty} s_{\sigma \mid k} \geq s\right\}\right) \geq s .
$$

Hence if $\liminf _{k \rightarrow \infty} s_{\sigma \mid k} \geq s$ for all except countably many $\sigma$ in $F$, then $\operatorname{dim}_{H}(F) \geq s$.

Proof. Let $\sigma \in\left\{\sigma \in\{1,2\}^{N}: \liminf _{k \rightarrow \infty} s_{\sigma \mid k} \geq s\right\}$. Then for a given natural number $m, s_{\sigma \mid k}>s-\frac{1}{m}$ for all but finite $k$. Thus

$$
\begin{aligned}
& h^{s-\frac{2}{m}}(\sigma) \\
& =\liminf _{k \rightarrow \infty}\left(c_{1}^{s-\frac{2}{m}}+c_{2}^{s-\frac{2}{m}}\right)\left(c_{\sigma \mid 1,1}^{s-\frac{2}{m}}+c_{\sigma \mid 1,2}^{s-\frac{2}{m}}\right)\left(c_{\sigma \mid 2,1}^{s-\frac{2}{m}}+c_{\sigma \mid 2,2}^{s-\frac{2}{m}}\right) \cdots\left(c_{\sigma \mid k, 1}^{s-\frac{2}{m}}+c_{\sigma \mid k, 2}^{s-\frac{2}{m}}\right) \\
& =\infty .
\end{aligned}
$$

Since $\mu_{t}\left(\left\{\sigma \in\{1,2\}^{N}: \liminf _{k \rightarrow \infty} s_{\sigma \mid k} \geq s\right\}\right)>0$ for any $t<s$, following the density theorem argument used in the proof of Theorem 1, we get $H^{s-\frac{2}{m}}(\{\sigma \in$ $\left.\left.\{1,2\}^{N}: \liminf _{k \rightarrow \infty} s_{\sigma \mid k} \geq s\right\}\right)=\infty$ for each $m$. Thus $\operatorname{dim}_{H}\left(\left\{\sigma \in\{1,2\}^{N}\right.\right.$ : $\left.\left.\liminf _{k \rightarrow \infty} s_{\sigma \mid k} \geq s\right\}\right) \geq s$.

Now assume that $\liminf _{k \rightarrow \infty} s_{\sigma \mid k} \geq s$ for all except countably many $\sigma$ in $F$. Noting that every countable subset of $F$ has $\mu_{t}$-measure 0 and $\mu_{t}(F)=1$ for each $t$, we easily see that $\operatorname{dim}_{H}(F) \geq s$.

In packing dimension cases, we get similar results. We list them as follows.

Theorem 5. If $\mu_{s}\left(\bigcup_{t>s} G_{t}\right)>0$, then $\operatorname{dim}_{p}\left(\bigcup_{t>s} G_{t}\right) \geq s$.

Corollary 6. $\operatorname{dim}_{p}(F) \geq \sup \left\{s>0: \mu_{s}\left(\bigcup_{t>s} G_{t}\right)>0\right\}$.

Corollary 7. $\operatorname{dim}_{p}\left(\bigcup_{t \leq s} G_{t}\right) \leq s$. 
Corollary 8. Let $s_{\tau}$ be the solution of the equation $c_{\tau, 1}^{s_{\tau}}+c_{\tau, 2}^{s_{\tau}}=1$. Then $\operatorname{dim}_{p}\left(\left\{\sigma \in\{1,2\}^{N}: \lim _{\sup _{k \rightarrow \infty}} s_{\sigma \mid k} \leq s\right\}\right) \leq s$. Hence $\operatorname{dim}_{p}(F) \leq s$ if $\lim \sup _{k \rightarrow \infty} s_{\sigma \mid k} \leq s$ for all except countably many $\sigma$ in $F$.

Proof. Let $\sigma \in\left\{\sigma \in\{1,2\}^{N}: \lim _{\sup _{k \rightarrow \infty}} s_{\sigma \mid k} \leq s\right\}$. Then for a given natural number $m, s_{\sigma \mid k}<s+\frac{1}{m}$ for all but finite $k$. Then

$$
\begin{aligned}
& q^{s+\frac{2}{m}}(\sigma) \\
& =\limsup _{k \rightarrow \infty}\left(c_{1}^{s+\frac{2}{m}}+c_{2}^{s+\frac{2}{m}}\right)\left(c_{\sigma \mid 1,1}^{s+\frac{2}{m}}+c_{\sigma \mid 1,2}^{s+\frac{2}{m}}\right)\left(c_{\sigma \mid 2,1}^{s+\frac{2}{m}}+c_{\sigma \mid 2,2}^{s+\frac{2}{m}}\right) \cdots\left(c_{\sigma \mid k, 1}^{s+\frac{2}{m}}+c_{\sigma \mid k, 2}^{s+\frac{2}{m}}\right) \\
& =0 .
\end{aligned}
$$

Following the dual density theorem argument related to packing measure used in the proof of Corollary 3, we get $p^{s+\frac{2}{m}}\left(\left\{\sigma \in\{1,2\}^{N}: \lim _{\sup _{k \rightarrow \infty}} s_{\sigma \mid k} \leq\right.\right.$ $s\})=0$ for each $m$. Thus $\operatorname{dim}_{p}\left(\left\{\sigma \in\{1,2\}^{N}: \lim \sup _{k \rightarrow \infty} s_{\sigma \mid k} \leq s\right\}\right) \leq s$.

Now assume that $\lim \sup _{k \rightarrow \infty} s_{\sigma \mid k} \leq s$ for all except countably many $\sigma$ in $F$. Noting that every countable subset of $F$ has $\mu_{t}$-measure 0 and $\mu_{t}(F)=1$ for each $t$, we easily see that $\operatorname{dim}_{p}(F) \leq s$.

Corollary $9([3])$. Let $s_{\tau}$ be the solution of $c_{\tau, 1}^{s_{\tau}}+c_{\tau, 2}^{s_{\tau}}=1$. If $\lim _{k \rightarrow \infty} s_{\sigma \mid k}=s$ for all $\sigma \in\{1,2\}^{N}$, then $\operatorname{dim}_{H}(F)=\operatorname{dim}_{p}(F)=s$.

\section{Application to Random Cantor Sets}

Consider a probability space $(\Omega, \mathcal{F}, P)$ (cf.[4]) such that the sample space

$$
\Omega=\left\{\left(c_{1}, c_{2}, c_{1,1}, c_{1,2}, c_{2,1}, c_{2,2}, c_{1,1,1}, \ldots, c_{\tau}, \ldots\right): 0<a \leq c_{\tau} \leq b<\frac{1}{2}\right\},
$$

and $\mathcal{F}=\sigma\left(\mathcal{F}_{1}, \mathcal{F}_{2}, \ldots, \mathcal{F}_{n}, \cdots\right)$, where

$$
\begin{aligned}
\mathcal{F}_{n}=\sigma(\{ & \left(\alpha_{1}, \beta_{1}\right] \times \cdots \times\left(\alpha_{2+\cdots+2^{n}}, \beta_{2+\cdots+2^{n}}\right] \\
& \left.\left.\times[a, b] \times[a, b] \times \cdots: a \leq \alpha_{i}<\beta_{i} \leq b\right\}\right) .
\end{aligned}
$$

We define $C_{\tau}(\omega)=c_{\tau}$ where $\omega=\left(c_{1}, c_{2}, c_{1,1}, c_{1,2}, c_{2,1}, c_{2,2}, c_{1,1,1}, \ldots, c_{\tau}, \ldots\right)$. We note that if $\omega \in \Omega$ is given, there corresponds a deranged Cantor set $F(\omega)$, where $F(\omega)$ is determined by the sequence of contraction ratios $\omega=$ $\left(c_{1}, c_{2}, c_{1,1}, c_{1,2}, c_{2,1}, c_{2,2}, c_{1,1,1}, \ldots, c_{\tau}, \ldots\right)$. We assume that $P$ is a probability measure on $(\Omega, \mathcal{F})$ such that $C_{j, 1}$ and $C_{j, 2}$ have the same distribution as $C_{i, 1}$ and $C_{i, 2}$ respectively where $j \in\{1,2\}^{n}$ and $i \in\{1\}^{n}, n=0,1,2, \ldots$

In particular, we note that Falconer([4]) assumed that $C_{j, 1}, C_{j, 2}$ have the same distribution as $C_{1}, C_{2}$ respectively for all $j$ and $C_{j}$ are independent random variables, except $C_{j, 1}$ and $C_{j, 2}$ for each $j$. Unlike Falconer, we now assume 
that $C_{j}$ are independent random variables except $C_{k}$ and $C_{l}$ for $k, l$ of the same length.

In this case we get some result about the packing dimension for the random Cantor set using the upper weak local dimensions.

Theorem 10. Let $C_{i, 1}=L_{n}$ and $C_{i, 2}=R_{n}$ for each $n=0,1,2, \ldots$ and $i \in\{1\}^{n-1}$. If we assume that for each $n, C_{j, 1}=L_{n}$ and $C_{j, 2}=R_{n}$ for all $j \in\{1,2\}^{n-1}$ and $E\left(L_{n}^{s}+R_{n}^{s}\right)=1$ for all $n$ for some $s$, then $\operatorname{dim}_{p}(F(\omega)) \leq s$ $P$-almost surely $\omega$. Further $p^{s}(F(\omega))<\infty$ P-almost surely $\omega$.

Proof. If the above condition is satisfied, the sequence of random variables $X_{n}\left(=\sum_{I \in E_{n}}|I|^{s}\right)$ forms an $L_{1}$-bounded martingale and hence converges to $X$ for $P$-almost all $F$. Since $E(X) \leq \liminf E\left(X_{n}\right)=1$ by Fatou lemma, $X<\infty, P$-almost surely. By Corollary 7 , it has packing dimension equal or less than $s$, where $E\left(L_{n}^{s}+R_{n}^{s}\right)=1$ for all $n$ for some $s P$-almost surely. In fact, we easily see that $p^{s}(F)<\infty P$-almost surely using the same arguments in the proof of the Corollary 7 .

\section{References}

[1] I. S. Baek, Dimensions of the perturbed Cantor set, Real Analysis Exchange, 19 No. 1 (1994), 269-273.

[2] I. S. Baek, On deranged Cantor sets, Kyungpook Math. Journal, 38 No. 2 (1998), 363-367.

[3] I. S. Baek, Dimensions of weakly convergent deranged Cantor set, Real Analysis Exchange, 23 No. 2 (1998), 689-696.

[4] K. J. Falconer, Fractal geometry, John Wiley and Sons. 1990.

[5] K. J. Falconer, Techniques in fractal geometry, John Wiley and Sons. 1997.

[6] C. Tricot, Two definitions of fractional dimension, Math. Proc. Camb. Phil. Soc., 91 (1982), 57-74. 